\title{
Detección de toxicidad subclínica por plomo en niños provenientes de la Cuenca Matanza Riachuelo
}

\section{Detection of subclinical lead toxicity in children from the Matanza Riachuelo Basin}

DOI: $10.46981 / \mathrm{sfjhv2n4-004}$

Received in: Oct 1st, 2021

Accepted in: Dec 31th, 2021

\author{
María Inés Galvagno \\ Médica Pediatra especialista en Medicina Interna Pediátrica \\ Hospital de Pediatría Prof. Dr Juan P. Garrahan \\ Av Caseros $24657^{\circ} \mathrm{E}, \mathrm{CABA}$, Argentina \\ E-mail: igalvagno78@gmail.com / inesuchi@live.com.ar \\ Mario Vallejo \\ Técnico de análisis clínico de laboratorio \\ Hospital de Pediatría Prof. Dr Juan P. Garrahan \\ Aranguren 1570, CABA, Argentina \\ E-mail: mariovallejo3000@gmail.com \\ Silvia Teresa Villafañe \\ Bioquímica Jefe de Área Laboratorio \\ Hospital de Pediatría Prof. Dr Juan P. Garrahan \\ Méndez de Andes $4775^{\circ} \mathrm{A}, \mathrm{CABA}$, Argentina \\ E-mail: silviavillaf@gmail.com \\ Daniel Enrique González \\ PhD Biochemistry-Toxicology \\ Hospital de Pediatría Prof. Dr Juan P. Garrahan \\ Viel 631, CABA, Argentina \\ E-mail:dgcasa@gmail.com \\ María Cristina Fernández \\ Consultora Salud Ambiental Infantil y Hospital Sostenible \\ Hospital de Pediatría Prof. Dr Juan P. Garrahan \\ Av Directorio $21673^{\circ} \mathrm{A}, \mathrm{CABA}$, Argentina \\ E-mail: mcfernan01@gmail.com
}

\section{RESUMEN}

Introducción: El plomo es un contaminante ambiental frecuente, y la exposición crónica a bajos niveles produce alteraciones en el área cognitiva y neuroconductual. La población más vulnerable es los niños entre 6 meses y 6 años. Actualmente, valores de plombemia (Pbs) mayores a $5 \mathrm{ug} / \mathrm{dl}$ requieren de una intervención. Sin embargo, no existe límite de seguridad en lo que se refiere a plombemia y neurotoxicidad.Población y Métodos: Se realizó un estudio prospectivo, descriptivo, aleatorizado en el Hospital Garrahan entre agosto de 2010 y abril de 2011, incluyéndose los pacientes que concurrieron espontáneamente a la consulta ambulatoria (Bajo y Mediano Riesgo) provenientes de la Cuenca Matanza 
Riachuelo, entre 1 y 6 años de edad. Resultados: Se incluyeron 237 pacientes, 2,7 años de edad promedio. Casi el 36\% de la población incluída presentó plombemias mayores a $5 \mathrm{ug} / \mathrm{dl}$. Del cuestionario surgió, como diferencia significativa entre los grupos, la existencia de algún trabajo de riesgo RR 3,9 (IC 95\% 1,7 a 8,6) p0.0004 (metalúrgicos, mecánicos, gasistas -litargirio-, recolección de chatarra). El 94\% presentó disminución significativa de la Pbs después de la intervención.

Conclusiones: La prevalencia de la población estudiada fue 36\% (n:85p) para valores mayores a 5ug/dl. Con el cumplimiento de las medidas higiénico-ambientales propuestas, se logró una disminución significativa de la $\mathrm{Pbs}$ de los niños expuestos. Identificar las fuentes ambientales potenciales e instar a su eliminación es de suma importancia en la consulta pediátrica.

Keywords: plombemia, intoxicación por plomo, plomo

\begin{abstract}
Introduction: Lead is a frequent environmental contaminant, and chronic exposure to low levels produces alterations in the cognitive and neurobehavioral areas. The most vulnerable population is children between 6 months and 6 years of age. Currently, plombemia values (Pbs) higher than $5 \mathrm{ug} / \mathrm{dl}$ require intervention. However, there is no safety limit regarding plombemia and neurotoxicity. Population and Methods: A prospective, descriptive, randomized, descriptive study was conducted at the Garrahan Hospital between August 2010 and April 2011, including patients who spontaneously attended the outpatient clinic (Low and Medium Risk) from the Matanza Riachuelo Basin, between 1 and 6 years of age. Results: We included 237 patients, average age 2.7 years. Almost $36 \%$ of the included population presented plombemia higher than $5 \mathrm{ug} / \mathrm{dl}$. From the questionnaire, a significant difference between the groups was the existence of some work at risk RR 3.9 (95\% CI 1.7 to 8.6) p0.0004 (metallurgists, mechanics, gas workers -litargirio-, scrap metal collection). Ninety-four percent presented a significant decrease in $\mathrm{Pbs}$ after the intervention. Conclusions: The prevalence of the population studied was $36 \%$ (n:85p) for values higher than $5 \mathrm{ug} / \mathrm{dl}$. With compliance with the proposed hygienic-environmental measures, a significant decrease in the Pbs of exposed children was achieved. Identifying potential environmental sources and urging their elimination is of utmost importance in the pediatric practice.
\end{abstract}

Keywords: plombemia, lead intoxication, lead, lead poisoning.

\title{
1 INTRODUCCIÓN
}

El plomo es un metal pesado que no desempeña ninguna función fisiológica en el humano, por lo que sus valores en plasma deberían ser indetectables. Se trata de un contaminante ambiental frecuente y representa un grave problema en la salud pública, especialmente si se tiene en cuenta que la población más vulnerable son los niños, trabajadores y personas de bajo nivel socioeconómico.

Las fuentes de exposición son: la industrial (baterías, cables, plásticos, vidrios, cristales, cañerías), la ambiental (fundición de plomo y otros metales y la emisión de plomo de la combustión de la nafta) y la doméstica (ingestión de pinturas que contienen plomo -hábito de pica-, cañerías de plomo que contaminan el agua potable). El vivir con personas expuestas ocupacionalmente al plomo, el bajo nivel socioeconómico, la presencia de cañerías de plomo en el hogar, la acumulación de polvo, la pintura 
descascarada, los pisos de tierra y la proximidad a industrias o depósitos de chatarra o baterías, son factores de riesgo para la exposición al plomo.

El plomo se absorbe a través de la vía gastrointestinal (las dietas pobres en hierro y calcio y ricas en lípidos aumentan la absorción de plomo) y respiratoria. Se distribuye en el sistema esquelético en donde la vida media es de 20 a 30 años y en tejidos blandos como riñón, sistema nervioso central e hígado. En la sangre se encuentra en los eritrocitos, siendo el principal compartimento responsable de la toxicidad.

El principal factor de riesgo para la intoxicación por plomo es la edad, siendo el pico entre los 18 y los 24 meses. Esto se debe a que los hábitos exploratorios de los niños aumentan la probabilidad de ingestión de polvo contaminado con plomo y también a que,en relación con la superficie corporal, las vías de absorción son mayores.

En cuanto a las manifestaciones clínicas encontramos, a nivel gastrointestinal, dolor abdominal y constipación. En el SNC, dado que el plomo atraviesa la barrera hematoencefálica, determina alteraciones permanentes en la arquitectura cerebral. Pueden presentar retraso mental, alteraciones del comportamiento, hiperactividad.

La intoxicación aguda es muy infrecuente, siendo la encefalopatía aguda la forma más común de presentación. La intoxicación crónica es la más frecuente y la sintomatología es inespecífica: alteraciones neuroconductuales, mal rendimiento escolar, constipación, náuseas, anorexia, Sindrome de Fanconi, anemia microcítica hipocrómica. Pueden observarse en radiografías de huesos largos los depósitos de plomo como líneas metafisarias, y un piqueteado lineal azulado en las encías (ribete de Burton).

Los trastornos de neurodesarrollo pueden inicialmente pasar inadvertidos y no ser relacionados con el tóxico ya que, en algunas ocasiones, los síntomas se manifiestan años después de la exposición al mismo. Este novedoso y especial comportamiento de ciertos tóxicos se reconoce como neurotoxicidad silente.

El tratamiento de la intoxicación se basa en: eliminación o reducción de la fuente de exposición, soporte nutricional y, de ser necesario, utilización de quelantes de plomo.

Existía con anterioridad a la realización de este trabajo, una clasificación propuesta en el año 1991 por los Centros para el Control de Enfermedades de Estados Unidos (CDC) según la cual se establecían recomendaciones para el seguimiento y el tratamiento de los pacientes según la plombemia. Se planteaba que con niveles mayores a $10 \mathrm{ug} / \mathrm{dl}$ se requería de alguna intervención.

En el año 2012 han recomendado disminuir el valor de referencia de 10 a 5 ug/dl.

Sin embargo, actualmente no existe límite de seguridad en lo que se refiere a plombemia y neurotoxicidad. 
El objetivo principal de este trabajo fue la detección de la toxicidad subclínica por plomo en los pacientes más vulnerables, por edad y por lugar de residencia (Cuenca Matanza Riachuelo) y, como objetivo secundario,detectar situaciones de riesgo a través de un cuestionario, realizar las intervenciones necesarias para disminuir la exposición al plomo y evaluar el impacto de las mismas.

\section{POBLACIÓN Y MÉTODOS}

Se realizó un estudio prospectivo, descriptivo, aleatorizado en el Hospital Garrahan entre agosto de 2010 y abril de 2011.

Se incluyeron los pacientes que concurrieron espontáneamente a la consulta ambulatoria (Bajo y Mediano Riesgo del Hospital Garrahan) provenientes de la Cuenca Matanza Riachuelo, los días hábiles de la semana, de 8 hs a 15 hs, con edades entre 1 y 6 años.

Se realizó una encuesta de riesgo ambiental (lugar de residencia, tipo de suministro de agua, ocupación de adultos convivientes, nivel de escolaridad del niño y de los padres y existencia de fábricas, talleres de auto, acopio de baterías en las cercanías al domicilio) y determinación de laboratorio (plombemia y hemograma). Los padres o tutores de los niños que se incluyeron en el trabajo firmaron un consentimiento informado.

Aquellos pacientes que presentaron plombemias mayores a $5 \mathrm{ug} / \mathrm{dl}$ fueron recitados a la consulta clínica, se dieron pautas para disminuir la exposición ambiental al plomo y se realizó nuevo dosaje de plomo.

A aquellos pacientes con plombemia mayor de $10 \mathrm{ug} / \mathrm{dl}$, se les realizó nueva plombemia a los 3 meses; y a los que tenían plombemia inicial entre 5 y $10 \mathrm{ug} / \mathrm{dl}$, a los 6 meses.

Para la determinación de plomo en sangre se utilizó un Espectrofotómetro de Absorción Atómica Varian AA240Z, con corrección Zeeman y los hemogramas se realizaron en contadores hematológicos Sysmex XE-2100 y microscopía óptica.

\section{RESULTADOS}

Participaron del estudio 237 pacientes. La edad promedio fue de 2,7 años (DS 1,4).

Al realizar el georreferenciamiento se observa que la mayoría de los pacientes proviene de La Matanza (33\%), Lomas de Zamora (15\%) y Merlo (15\%).

El $9 \%$ de los niños $(n=22 p)$ presentó plombemia inicial mayor de $10 \mathrm{ug} / \mathrm{dl}, 27 \%(\mathrm{n}=63 \mathrm{p})$ entre 5 y $10 \mathrm{ug} / \mathrm{dly}$ el $64 \%$ menor de $5 \mathrm{ug} / \mathrm{dl}(\mathrm{n}=152 \mathrm{p})$ 
Ilustración 1. Plombemia inicial

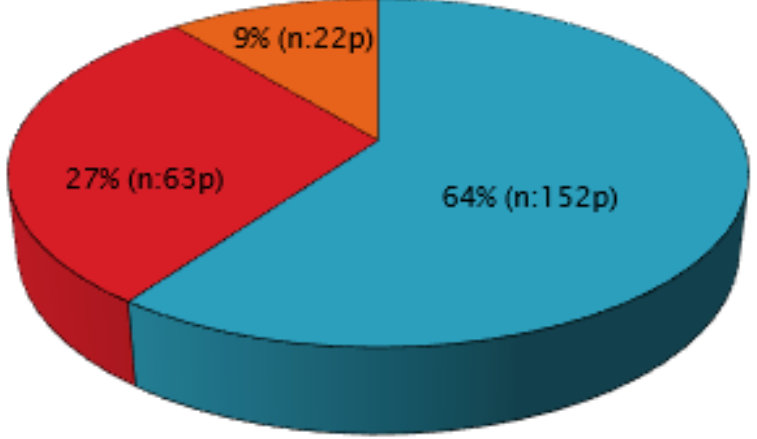

MENOR A 5

5 A 10

$\square$ MAYOR A 10

Ilustración 2. Variables analizadas en los diferentes grupos

\begin{tabular}{|l|l|l|l|l|}
\hline $\begin{array}{l}\text { Valores Pb } \\
\mathrm{n}\end{array}$ & $\begin{array}{l}\text { Menor 5 } \\
152\end{array}$ & $\begin{array}{l}\mathbf{5} \text { a 10 } \\
63\end{array}$ & $\begin{array}{l}\text { Más de 10 } \\
22\end{array}$ & \\
\hline Sexo masculino & $65(43 \%)$ & $28(44 \%)$ & $11(50 \%)$ & NS \\
\hline Edad & $2,9 \pm 1,4$ & $2,3 \pm 1,2$ & $2,5 \pm 1,3$ & NS \\
\hline Vivienda rural & $4(3 \%)$ & $2(3 \%)$ & 0 & NS \\
\hline Agua de pozo & $70(46 \%)$ & $24(38 \%)$ & $10(45 \%)$ & NS \\
\hline Fábrica cercana & $110(72 \%)$ & $51(81 \%)$ & $17(77 \%)$ & NS \\
\hline Trabajo de riesgo & $37(24 \%)$ & $14(22 \%)$ & $13(59 \%)$ & 0,0002 \\
\hline
\end{tabular}

De los datos obtenidos se desprende que no existen diferencias significativas entre los diferentes grupos en las variables analizadas, excepto el antecedente de existir en la familia algúntrabajo de riesgo(metalúrgicos, mecánicos, gasistas -litargirio-, recolección de chatarra)

Ilustración 3. Plombemias iniciales y post intervención

\begin{tabular}{|l|l|l|l|l|}
\hline & $\begin{array}{l}\text { Grupo de } \\
\text { pacientes }\end{array}$ & Pb inicial & $\begin{array}{l}\text { Pb post } \\
\text { intervención }\end{array}$ & p \\
\hline $\begin{array}{l}\text { Mediana } \\
\text { Pc 25-75 }\end{array}$ & $\begin{array}{l}\text { Pb entre 5 y } 10 \\
\text { ug/dl }\end{array}$ & $\frac{6,9 \mathrm{ug} / \mathrm{dl}}{(5,6-8)}$ & $\frac{4 \mathrm{ug} / \mathrm{dl}}{(4-4)}$ \\
& $\begin{array}{l}\text { Pb mayor de } 10 \\
\text { ug/dl controles })\end{array}$ & $<0,0001$ \\
\hline & $\frac{12,1 \mathrm{ug} / \mathrm{dl}}{(10,8-15,2)}$ & $\begin{array}{l}\frac{4,5 \mathrm{ug} / \mathrm{dl}}{(4-5,7)} \\
(18 \text { controles })\end{array}$ & 0,0003 \\
\hline
\end{tabular}

\subsection{RESULTADOS DE LOS PACIENTES RECITADOS, POR GRUPOS:}

* Plombemia inicial entre 5 y $10 \mathrm{ug} / \mathrm{dl}$ : de los 63 pacientes pertenecientes a este grupo, 27p $(42,9 \%)$ realizaron el control correspondiente de los 6 meses.

El 96,3\% (26p) presentaron una disminución de la plombemia, el 80,8\%(21p) a valores normales (menor de $5 \mathrm{ug} / \mathrm{dl}$ ) y el 19,2\% (5p) a valores entre 5 y $10 \mathrm{ug} / \mathrm{dl}$ (con una mediana de $5,7 \mathrm{ug} / \mathrm{dl}$ ).

Sólo un paciente presentó un aumento de la plombemia, manteniéndose con valor entre 5 y 10 ug/dl. 
* Plombemia inicial mayor de $10 \mathrm{ud} / \mathrm{dl}$ : de los 22 pacientes, 18p $(81,8 \%)$ realizaron el control correspondiente de los 3 meses.

El 94,4\% (17p) presentaron una disminución de la plombemia, el 52,9\% (9p) a valores normales y el $41,2 \%$ (7p) a valores entre 5 y $10 \mathrm{ug} / \mathrm{dl}$ (con una mediana de $5,7 \mathrm{ug} / \mathrm{dl}$ ).

Un paciente se mantuvo sin modificaciones en la plombemia de control.

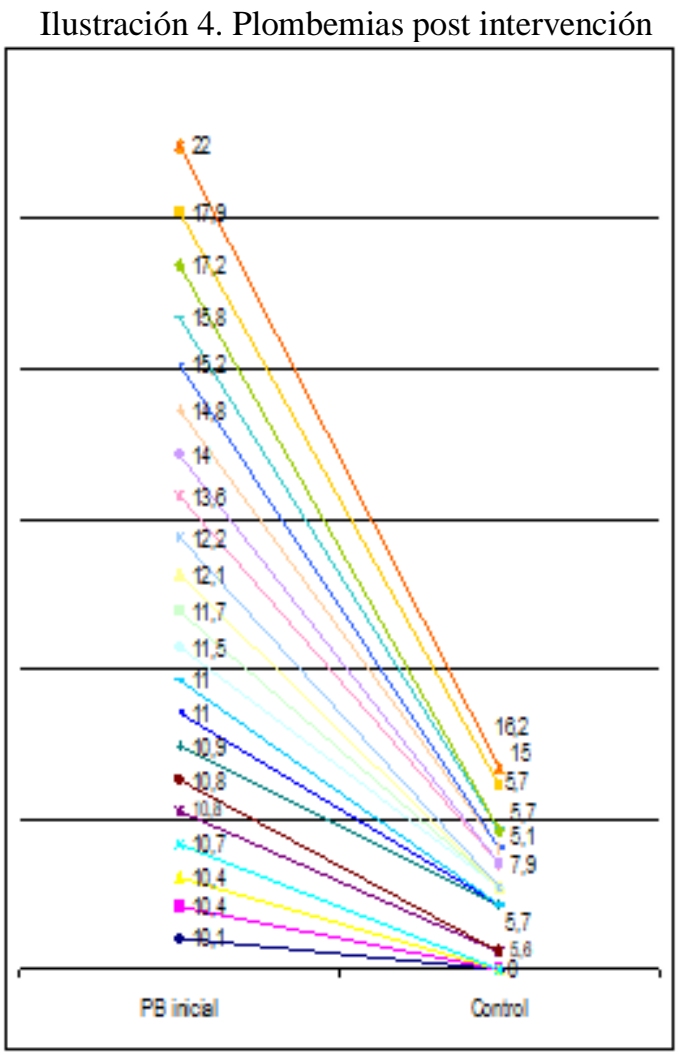

\section{DISCUSIÓN}

La cuenca del río Matanza-Riachuelo abarca aproximadamente $2.200 \mathrm{~km}^{2}$, y se conforma por una parte de la Ciudad Autónoma de Buenos Aires y 14 partidos de la provincia de Buenos Aires: Lanús, Avellaneda, Lomas de Zamora, Esteban Echeverría, La Matanza, Ezeiza, Cañuelas, Almirante Brown, Morón, Merlo, Marcos Paz, Presidente Perón, San Vicente y General Las Heras. Discurren los 64 km de extensión del sistema hídrico integrado por los ríos Matanza y Riachuelo. Se trata de un territorio que cuenta con una alta densidad poblacional y niveles elevados de contaminación ambiental, siendo la cuenca fluvial más contaminada de Argentina.

Entre las principales causas de contaminación de la Cuenca Matanza Riachuelo se encuentra la inadecuada gestión de los residuos. Los basurales a cielo abierto son lugares donde los residuos urbanos son dispuestos sin ningún tipo de tratamiento ni medidas de seguridad. La disposición final de los residuos bajo esta modalidad fue una práctica común durante muchos años a lo largo de todo el país, resultando en todos los casos en distintos problemas ambientales. La política de erradicación aplicada en los últimos años logró reducir estos sitios, pero aún continúa siendo una problemática para la región. 
Se sabe que la toxicidad depende de la edad del paciente, del período del desarrollo, del tipo y dosis del tóxico.Puede afectar al sistema nervioso en forma insidiosa, produciendo síntomas inespecíficos como cambios del estado de ánimo, fatiga y disfunción cognitiva.

Hace pocos años se comenzó a profundizar en el conocimiento de que la exposición a sustancias tóxicas ambientales puede provocar alteraciones en el neurodesarrollo y en la salud.

Los efectos observados se relacionan con el momento de exposición, ventanas de vulnerabilidad en períodos críticos del desarrollo (organogénesis e histogénesis del cerebro) que se extiende desde la etapa embrionaria a la adolescencia;con el tiempo de exposición y con la sensibilidad individual, afectando el neurodesarrollo de manera específica y permanente.

En los últimos años hubo un llamativo aumento en el diagnóstico de trastorno por déficit de atención con hiperactividad y del trastorno generalizado del desarrollo.

La asociación de estos trastornos del desarrollo con entidades genéticas neurológicas es baja. Factores epigenéticos y ambientales pueden contribuir a este llamativo incremento, así como a la variable expresividad de los trastornos.

En el abordaje y seguimiento de pacientes con dichos diagnósticos, y en toda consulta pediátrica en general, se deberá poder pesquisar de manera temprana la exposición a tóxicos ambientales y poder realizar una intervención temprana y adecuada.

\section{CONCLUSIONES}

Luego de detectar aquellos pacientes expuestos al plomo y tras la intervención a través de la entrevista con los familiares, se logró una franca disminución de la plombemia de los niños expuestos. Detectar la posible fuente de exposición y evaluar junto a los padres las diferentes posibilidades para poder eliminarlas, fue de suma importancia en todos los casos para poder lograrlo.

Consideramos de suma importancia la detección de las situaciones de riesgo, particulares de cada uno de nuestros pacientes, a fin de poder orientarlos e intervenir con el objetivo de disminuir la exposición a este tóxico ambiental.

Observamos en este trabajo que los hábitos y los trabajos de riesgo que exponen a los pacientes y familiares al plomo son los principales relacionados con los valores más altos de plombemia. Resulta fundamental y perentorio incorporar a nuestra práctica médica, la sospecha y la pesquisa temprana de estas situaciones, a fin de poder realizar las intervenciones necesarias.

Las entrevistas realizadas, la concientización acerca de esta problemática, la adherencia a las sugerencias realizadas para disminuir la fuente de exposición, fueron fundamentales en este trabajo para poder lograr la disminución de la plombemia de los niños expuestos. 


\section{BIBLIOGRAFÍA}

- Dr. A. Ignacio Ascione, Intoxicación por plomo en Pediatría, Arch Pediatr Urug 2001; 72(2): 133-138.

- Martins, E., Varea, A., Hernández, K., Sala, M., Girardelli, A., Fasano, V., \& Disalvo, L. (2016). Blood lead levels in children aged between 1 and 6 years old in La Plata, Argentina. Identification of risk factors for lead exposure. Niveles de plombemia en niños de 1 a 6 años en la región de La Plata, Argentina. Identificación de factores de riesgo de exposición al plomo. Archivos argentinos de pediatría, 114(6), 543-549. https://doi.org/10.5546/aap.2016.eng.543.

- Ministerio de Salud de la Nación. Programa Nacional de Prevención y Control de Intoxicaciones Precotox 2013 Guía De Prevención, Diagnóstico, Tratamiento Y Vigilancia Epidemiológica De Las Intoxicaciones Ambientales Infantiles con Plomo.

- Hugo A. Arroyo, María Cristina Fernández.Tóxicos ambientales y su efecto sobre el neurodesarrollo.MEDICINA (Buenos Aires) 2013; 73 (Supl. I): 93-102.

- Intoxicación e indicadores de efecto y exposición a agentes tóxicos. Normativa y tutorial para la vigilancia a través del Sistema Nacional de Vigilancia en Salud -SNVS- 2014.

- Millan M. J. (2013). An epigenetic framework for neurodevelopmental disorders: from pathogenesis to potential therapy. Neuropharmacology, 68, 2-82. https://doi.org/10.1016/j.neuropharm.2012.11.015.

- National Research Council (US) Committee on Developmental Toxicology. (2000). Scientific Frontiers in Developmental Toxicology and Risk Assessment. National Academies Press (US). 